\title{
Article
}

\section{Examining the visual processing patterns of lonely adults}

Bangee, Munirah and Qualter, Pamela

Available at http://clok.uclan.ac.uk/21874/

Bangee, Munirah ORCID: 0000-0001-8548-6692 and Qualter, Pamela (2018)

Examining the visual processing patterns of lonely adults. Scandinavian Journal of Psychology, 59 (4). pp. 351-359. ISSN 0036-5564

It is advisable to refer to the publisher's version if you intend to cite from the work. http://dx.doi.org/10.1111/sjop.12436

For more information about UCLan's research in this area go to

http://www.uclan.ac.uk/researchgroups/ and search for < name of research Group>.

For information about Research generally at UCLan please go to http://www.uclan.ac.uk/research/

All outputs in CLoK are protected by Intellectual Property Rights law, including Copyright law. Copyright, IPR and Moral Rights for the works on this site are retained by the individual authors and/or other copyright owners. Terms and conditions for use of this material are defined in the policies page.

\section{CLoK}

Central Lancashire online Knowledge www.clok.uclan.ac.uk 
Examining the visual processing patterns of lonely adults 


\begin{abstract}
Prior research has shown that loneliness is associated with hypervigilance to social threats, with eye-tracking research showing lonely people display a specific attentional bias when viewing social rejection and social exclusion video footage (Bangee, Harris, Bridges, Rotenberg \& Qualter, 2014; Qualter et al., 2013). The current study uses eye-tracker methodology to examine whether that attentional bias extends to negative emotional faces and negative social non-rejecting stimuli, or whether it could be explained only as specific bias to social rejection/exclusion. It is important to establish whether loneliness relates to a specific or general attention bias because it might explain the maintenance of loneliness. Participants $(\mathrm{N}=43, \mathrm{~F}=35$, Mage $=20$ years and 2 months, $\mathrm{SD}=3$ months $)$ took part in 3 tasks, where they viewed different social information: Task 1- slides displaying 4 faces each with different emotions (anger, afraid, happy and neutral), Task 2 - slides displaying 16 faces with varying ratios of happiness and anger, and Task 3 - slides displaying 4 visual scenes (socially rejecting, physically threatening, socially positive, neutral). For all three tasks, eye movements were recorded in real time with an eye-tracker. Results showed no association between loneliness and viewing patterns of facial expressions, but an association between loneliness and hypervigilant viewing of social rejecting stimuli. The findings indicate that lonely adults do not have a generalised hypervigilance to social threat, but have, instead, a specific attentional bias to rejection information in social contexts. Implications of the findings for interventions are discussed.
\end{abstract}

Keywords: Loneliness; attention; cognitive bias; eye-tracker; hypervigilance 


\section{Introduction}

Loneliness is an unpleasant feeling caused by a perceived discrepancy between the social relationships an individual currently has and those he/she wishes to have (Peplau \& Perlman, 1982). Loneliness is seen to be an inevitable part of life (Cacioppo \& Hawkley, 2009), with episodes of loneliness being normal and necessary for survival because they ensure we strengthen our social connections and keep ourselves safe from the dangers of living on the social perimeter. The beneficial aspects of loneliness diminish when it is intense and prolonged - when it becomes chronic loneliness - because it leads to a variety of shortand long-term negative health effects (Holt-Lunstad, Robles, \& Sbarra, 2017). Given those negative effects, there has been increased demand to understand the mechanisms involved in the maintenance of chronic loneliness and what cognitive processes might be implicated.

One model postulates that when people become lonely they shift their attention so they are attentive to social threats in the social environment, arguing that over time, this hypervigilance may undermine the opportunity to develop positive social relationships (Cacioppo \& Hawkley, 2009). However, little research has directly examined the attention processes of lonely people and determined whether attention to social threats is linked to social threats in general (i.e. negative emotional information in faces and social situations) or to specific social threats (i.e. socially rejecting cues). Knowing how attention processing is linked to loneliness, and how it might, if used over-time, maintain feelings of loneliness, will help in the development of interventions designed to mitigate loneliness.

\section{Loneliness and Perception}

Lonely adults show heightened attention to negative social words (Egidi, Shintel, Nusbaum \& Cacioppo, 2008), greater recall of social events in general (Gardner, Pickett, Jefferis \& Knowles, 2005), and perform worse on social monitoring tasks that are framed to test their social skills (Knowles, Lucas, Baumeister \& Gardner, 2015). Lonely people also 
interpret social situations as more threatening (Cacioppo et al, 2000; Jones Freemon \& Goswick, 1981), report higher levels of interpersonal stress (Doane \& Adam, 2010), and make more self-derogatory attributions (Snodgrass, 1987) in comparison to non-lonely people.

Loneliness is also reflected in the way the brain processes visually presented social information. In an early fMRI study, Cacioppo, Norris, Decety, Monteleone and Nusbaum (2009) found that lonely people have greater activation in the visual cortex when viewing unpleasant social pictures of people than objects. Two recent EEG studies extended that work and demonstrated that lonely people detect and process social threat more quickly than nonlonely people when tasks were presented using words (Cacioppo, Balogh \& Cacioppo, 2015) and images (Cacioppo, Bangee, et al, 2016). Such findings suggest that lonely people show automatic and implicit hypervigilance to social threats in the brain.

A specific brain structure has also been linked to loneliness, with Kanai et al (2012) finding that lonely people have reduced grey matter in the left posterior superior temporal sulcus (pSTS), an area involved in biological motion and social perception. The pSTS has been implicated in basic social perception skills and processing of social information, and Kanai et al., found that loneliness and pSTS size were related to poorer performance on gaze perception during four social perception tasks.

Attention allocation has further been examined using eye-tracking techniques. In each study, participants' eye movements were recorded, but the stimuli were different between studies: in Lodder et al. (2015), participants were presented with 4 images, one in each of the four quadrants of the computer screen (Task 2 anger, fear, happiness and neutral expression; Task 3: positive and negative social and non-social images), or with video clips that showed examples of positive or negative emotions (Task 4); in Bangee et al (2014) and Qualter et al. (2013) participants were shown short video clips that included examples of both 
positive social engagement between individuals and some element of social exclusion/rejection in another part of the screen. There is evidence from the Bangee et al. and Qualter et al. studies that loneliness increased hypervigilance towards the parts of the video that showed social exclusion, but loneliness was not associated with specific attention processing patterns towards the still images or video clips in the Lodder et al. study. We argue that the difference in findings is either (1) an outcome of the fact that Bangee et al. and Qualter et al. used stimuli focused on social exclusion/rejection while the Lodder et al. did not, or (2) to do with the fact that participants viewed dynamic images of both positive and negative social interactions at the same time in the Bangee et al. and Qualter et al., studies. Taken together, those findings highlight the need to examine whether still images of social exclusion/rejection elicit the same pattern of hypervigilance attention processing as found in Bangee et al. and Qualter et al. Findings will help ascertain whether the pattern of bias attention found amongst lonely people is characterised by hypervigilance to social rejection in the environment.

Negative emotions may also signal rejection (Burklund, Eisenberger, \& Lieberman, 2007), and, thus, could be seen as socially threatening. Thus, accuracy at identifying negative emotions and sensitivity towards negative emotions in faces has been examined. Studies exploring emotion recognition of negative vs. positive emotions showed that lonely adolescents were quicker to correctly identify sad and fearful emotional faces when completing an emotional recognition task that used video footage (Vanhalst, Gibb, \& Prinstein, 2015), and accurately recognized angry faces during emotional recognition tasks (Lodder et al., 2016); both studies found that loneliness was not related to the accuracy or sensitivity towards positive emotions. Spithoven, Bijttebier, \& Goossens, (2017) in their recent review of loneliness and cognitive processes note that those results are in line with the evolutionary theory of loneliness (Cacioppo \& Hawkley, 2009; Qualter et al., 2015) that 
posits loneliness is related to increased recognition and sensitivity for negative emotions. In the current study, we extend that research to understand whether the cognitive bias exhibited by lonely people generalises to negative facial expressions as well as being linked to other negative social information, or whether, instead, it is linked only to certain types of negative social cues (i.e. rejection cues). Knowing whether lonely people are on high alert for certain types of social threats is important because such knowledge will help in the development of targeted interventions to reduce loneliness. In the current study, as well as examine the attention processing of emotional faces as images in four quadrants of the screen, we also use the Face in The Crowd paradigm to examine whether people scoring higher on loneliness are sensitive to the angry faces in the crowd. Thus, we extend the work on emotion recognition and loneliness still further to examine the detection of anger - and thus, social danger - when it is hidden among a crowd of happy faces.

\section{Interplay of Related Constructs with Loneliness}

Social anxiety is highly related to loneliness and a meta-analysis reported medium effect sizes between the two constructs (Mahon, Yarcheski, Yarcheski, Cannella \& Hanks, 2006). More recently, Lim, Rodebaugh, Zyphur \& Gleeson (2016) argued there are strong links between social anxiety and loneliness because they share an important common cognitive feature, i.e. hypervigilance to social threat. Similarly, depression is strongly correlated with loneliness $(r=.40$ to .60$)$, with many researchers viewing loneliness as a symptom of depression, as evidenced by the inclusion of loneliness items (i.e., I felt lonely) on questionnaire measures for depression (i.e., Radloff, 1977). However, it has been argued that social anxiety (e.g. Jones, Rose \& Russell, 1990) and depression (e.g. Cacioppo, Hawkley \& Thisted, 2010; Lasgaard, Goossens \& Elklit, 2011) are constructs distinct from loneliness. Recently, researchers have emphasised the need to statistically control for these two constructs when examining loneliness (e.g. Lodder et al, 2015; Vanhalst et al, 2015), 
proposing that, because loneliness is a core feature of both social anxiety and depression, removing its variance in analyses leaves an examination of "pure" loneliness. While one may wonder what aspect of loneliness we are left with when we remove the key shared component of negative affect, in the current study, social anxiety and depressive symptoms are important to control because they, too, have been previously linked to hypervigilance to social threat using eye-tracker methodology (e.g. Armstrong \& Olatunji, 2012; Lim et al., 2016).

\section{Related Constructs and Eye-Tracking Research}

Eye-tracking studies (Bangee et al., 2014; Qualter et al., 2013) suggest that lonely people display attentional biases that reflect hypervigilance towards social rejection cues when viewing real-life video footage. Lonely children show disengagement difficulties when viewing socially rejecting stimuli (Qualter et al., 2013), whilst lonely young adults show an initial vigilance to socially rejection stimuli followed by avoidance (Bangee et al, 2014). These types of attention biases displayed by lonely people reflect similar patterns of processing found in the social anxiety and depression literature. Socially anxious adults show one of two strategies when viewing social threat stimuli: (1) they initially direct their attention towards the threat stimuli followed by attentional avoidance of the same stimuli (i.e. vigilance-avoidance hypothesis; Mogg, Bradley, de Bono \& Painter, 1997; Garner, Mogg \& Bradley, 2006), or (2) they show disengagement difficulties from threat cues, thus, are unable to disengage their attention efficiently (Fox, Russo \& Dutton, 2002; Schofield, Johnson, Inhoff \& Coles, 2012). These different patterns of processing found among socially anxious groups may be due to dissimilar methodologies or the use of different cut off scores for social anxiety. Elsewhere (Qualter et al., 2015), we have proposed that these different patterns among socially anxious individuals are also replicated among lonely groups and may represent age related changes in attention processing. Adults with depression symptomology show impairment in disengaging from negative emotional information (Caseras, Garner, 
Bradley \& Mogg, 2007; Sears, Thomas, LeHaquet \& Johnson, 2010). Such findings show the necessity of controlling for social anxiety and depression in eye-tracking studies examining loneliness and hypervigilance to social threat.

The eye-tracker is particularly useful to assess both early and late processing of attention continuously. It is a fast and continuous measure of attention, and is not contingent on a key press to measure attention. There are different components of attention processing to social threat stimuli: (1) initial vigilance and maintenance (i.e. hypervigilance) relates to the speed of attention to threat (Armstrong \& Olatunji, 2012), (2) disengagement difficulties refers to attention being captured by the threat stimuli (Buckner, Maner, \& Schmidt, 2010), and (3) attentional avoidance refers to orienting attention away from threat (Lange et al., 2011). The initial vigilance and maintenance pattern of processing is thought to be automatic, unintentional and outside voluntary control, while the latter attention processes (attentional avoidance, disengagement difficulties) is thought to be strategic, intentional, under voluntary control and occur on a later timescale during extended viewing (Cisler \& Koster, 2010).

Based on these distinctions between components of attention, previous eye-tracker studies (i.e. Hermans, Vansteenwegen \& Eelen, 1999) recommend the use of time-blocks to assess the patterns of attention deployment. Initial vigilance is demonstrated in the first 1000 milliseconds and attentional avoidance or disengagement difficulties demonstrated in the next 2000 milliseconds to the social threatening stimuli. The two eye-tracking studies (Bangee et al, 2014; Qualter et al, 2013) that examined loneliness and hypervigilance to social rejection used time-blocks. However, the study conducted by Lodder et al, (2015) that examined loneliness on emotional expressions did not report any findings using time-blocks to assess attention deployment.

Most of the eye-tracking research has used static images from a number of databases (International Affective Picture System; IAPS, Karolinska Directed Emotional Faces; KDEF, 
NimStim facial stimuli set), which has been previously validated. Static images are useful because they provide consistent evidence of how individuals process social information and cues in the environment. Even though the use of static images has been criticised (e.g. proxy measure; Bogels \& Mansell, 2004), they have been equivocally and consistently used in research. The current study uses (1) images of emotional faces, four being presented at once on screen, (2) the angry face-in-the happy crowd paradigm, and (3) images of complex social scenes, again with four images appearing on screen at once. As noted earlier, we propose, in line with other researchers (Spithoven et al., 2017) that negative facial emotions could be viewed as signals of rejection, meaning that both the facial and actual social scenes could be viewed as depictions of social rejection. Thus, for Task 1 and 3, we expected, based on previous work, for loneliness to be associated with attention to the socially threatening stimuli either in the form of angry faces (Task 1) or peer rejection/exclusion (Task 3). The stimuli in Task 2 - the angry face in the crowd paradigm - is used to explore whether loneliness increased the detection of anger, a potential sign of social rejection. In Task 2, we focused on the detection of anger because its detection would indicate a rapid detection of danger in the environment, supporting the current model of loneliness (Cacioppo \& Hawkley, 2009; Qualter et al., 2015). In Task 2, we also made the decision not to use neutral faces given recent discussion that people may be unbiased when viewing neutral stimuli, assigning negative or positive emotions to neutral faces based on previous knowledge (see Lazerus, Ingbretsen, Stolier, Freeman, \& Cikara, 2016; Suess Rabovsky, \& Rahman, 2015); evidence already exists showing that loneliness is associated with the assignment of hostility to ambiguously motivated social exclusion (Qualter et al., 2013) and we wanted to avoid tapping into that effect.

Using these three tasks and eye-tracker methodology, we examined whether people scoring higher on loneliness had an attention bias to social threat that represents a generalised 
bias to all social threats (i.e. all negative facial expressions and negative social scenes) or a specific bias to certain types of threats (i.e. those images depicting social rejection). In the analyses, we statistically control for social anxiety and depressive symptoms because of their known overlap with loneliness and cognitive biases. We also screened participants before invitation to the laboratory to complete the tasks so we could invite people scoring in top third of loneliness scores, matching them with participants who did not score in upper third of potential loneliness scores; this approach enabled us to ensure we had a sufficient number of people in our sample who reported high levels of loneliness. We predicted that loneliness would be associated with attention to negative emotional expressions and hypervigilance in the early stages of visual processing for social rejection stimuli. The specific aims of each eye-tracker task are as follows:

(1) Task 1 examined whether lonely adults showed hypervigilance in the early stages of processing to social threat information when depicted as negative emotional facial expressions (e.g. angry).

(2) Task 2 examined whether lonely adults showed attention to social threats depicted as angry faces in a group context (e.g. when angry and happy facial expressions are presented in a group of faces).

(3) Task 3 examined whether lonely adults show hypervigilance in the early stages of processing social threats that are depicted as static visual scenes showing instances of social rejection or social exclusion, alongside other negative social scenes and positive images.

\section{Method}

\section{Participants}

43 adults (8 males and 35 females) from a university in North West of England, UK, took part in the current study. The Ethics committee at the University of Central Lancashire, UK, approved the study. Participants were students and staff at the University, recruited by 
posters and the internal online recruitment system. Potential study participants were asked to complete an online pre-screening loneliness measure and extreme loneliness scorers at the high end of spectrum were invited to take part in the eye-tracker tasks; participants scoring within the other range of scores were not all selected to take part in the study, with only those who were matched according to gender and age with a participant high on loneliness taking part. 60 people completed the study on-line, with 56 being called to take part in the eyetracking studies, and 55 completing the tasks. The mean age of participants who took part in the eye-tracker tasks and whose data are presented here was 20 years and 2 months $(\mathrm{SD}=3$ months). The age range was restricted to between 18 and 30 years because prior evidence suggests that age impacts cognitive ability in cognitive tasks (Ebner, He \& Johnson, 2011; Hartshorne \& Germine, 2015; Verhaeghen \& Salthouse, 1997) and performance on eyemovements tasks (Munoz, Broughton, Goldring and Armstrong, 1998). Data for 12 participants were not used in the reported analyses because they were above the age range of the study. For Task 2, data from one participant could not be included in the analyses because their data were not recorded due to technical errors. The same sample of participants took part in all three tasks. No participants were excluded from the eye-tracker tasks based on visual acuity.

\section{Measures}

Loneliness. Loneliness was measured using The UCLA Loneliness scale (University of California, Los Angeles; Russell, 1996). This comprises 20 questionnaire items, including 'How often do you feel you are no longer close to anyone?' and 'How often do you feel left out?' Participants indicated how often they felt the way described in each statement on a 4point scale (Never, Rarely, Sometimes, Often). The measure is highly reliable, with internal consistency ranging from $\alpha=.89$ to .94 and test-retest reliability over a 1-year period being $\mathrm{r}$ $=.73$, and exhibits significant construct validity with measures of individual's interpersonal 
relationships, and measures of health and well-being (Russell, 1996). The scores for the scale range from 20 to 80 , with scores ranging from 24 to 73 in the current set of study. Mean scores were used in the analyses, with higher scores indicating higher levels of loneliness. A loneliness score of 60 or above was used to classify participants as members of the lonely group. In the current sample the scale exhibited excellent internal consistency $\alpha=.95$.

Social anxiety. The Brief Fear of Negative Evaluation Scale (BFNE; Leary, 1983a) and Interaction Anxiousness Scale (IAS; Leary, 1983b; Leary \& Knowalski, 1993) assessed levels of social anxiety. The BFNE includes 12 questions and the IAS comprises 15 questions; participants rated each statement on how characteristic it is of them using a 5-point scale $(1=$ not at all to $5=$ extremely) for both scales. The BFNE is highly reliable (internal consistency: $\alpha=.97$; test-retest reliability over a 2-week period: $r=.94$ ) and exhibits significant construct validity with measures of social avoidance and depression (Leary, 1983a). The IAS indicated high test-retest and internal reliability. Significant construct validity was shown with measures of social and general anxiety and with measures of anxiety and interpersonal concern in actual interactions (Leary \& Knowalski, 1993). Higher scores indicated higher levels of social anxiety. In the current sample, the scales exhibited good internal consistency $\alpha=.91$ and $\alpha=.88$, respectively. Social anxiety was assessed using two questionnaire measures because both fear of negative evaluation and an anxiousness to interact with others are core features of social anxiety and either/both of these features could be related to loneliness. Thus, both measures were used as assessment measures in order to control for the correct features of social anxiety in the analyses.

Depression. The Centre for Epidemiologic Studies Depression Scale (CES-D; Rudloff, 1977) was used to assess depressive symptomology. The scale included 20 questions requiring participants to indicate how often they felt the way described in the past week from four possible options (Rarely or none of the time; some or a little of the time; occasionally or 
a moderate amount of time; most or all of a time). The CES-D is has good reliability (internal consistency: $\alpha=.85$ to .90 ; test-retest reliability over a 8 -week period: $r=.59$ ) and exhibits significant construct validity with clinical criteria and self-reported measures (Rudloff, 1977). In the current study the item 'I felt lonely' was removed from the total score. Scores were summed and higher scores indicated higher levels of depressive symptoms. In the current sample, the scale exhibited excellent internal consistency $\alpha=.92$.

\section{Eye-Tracking Device}

Eyelink II model (with monocular recording at 500Hz) was used to track precise eye movements and foveal fixations for each participant in each task. Data viewer was used to record eye movements and monitor the specified areas of interest. Attention was operationalized in terms of eye fixations. An eye fixation was recorded whenever the participant stopped or had a saccade in any of the areas of interest that were previously coded in the software. A fixation occurrence was determined according to a standard logarithm of at least $100 \mathrm{~ms}$ in a given radius of 0.5 degrees of visual angle.

\section{Procedure}

Participants from a range of scores on loneliness were invited to take part in the series of eye-tracker tasks. Participants read the study information sheet before they completed the on-line pre-screening questionnaires, with consent provided by completing the questionnaires on-line. On the day they visited the experimental room at the university to complete the measures again and to take part in the eye-tracker tasks, participants re-read the information sheet and gave verbal consent to take part. Participants' scores on the questionnaires on the day, rather than those completed online, were used in the analyses. Participants then took part in the three eye-tracker tasks where they viewed three different picture tasks like they would to do so when watching television. Eye responses (initial fixations, time spent on each picture) were recorded with the eye-tracker technology in real time. The three different 
picture tasks were counterbalanced for the participants; the eye-tracker was calibrated for each task per participant.

Eye-tracker Task 1. Emotional facial stimuli were selected from the Karolinska Directed Emotional Faces database (KDEF; Lundqvist, Flykt \& Öhman, 1998). The database contains a valid set of affective facial pictures (Goeleven et al. 2008). Four emotional expressions of the same person expressing happy, angry, afraid and neutral emotions were presented at the same time. The angry and fearful emotional expressions were selected because those emotions are classed as socially threatening cues. The facial expression depicting happiness was chosen to reflect a positive emotion, while the neutral expression was chosen because it reflects a non-emotional state. A 2 by 2 matrix was used to present the four emotional expressions simultaneously to participants. Each matrix of pictures was randomized such that any of the four emotional expressions could appear in any location within the 2 by 2 matrix (i.e. top left, top right, bottom left, bottom right). This randomization strategy increases the reliability and makes for a stronger design of the overall study. In total 24 pictures slides were created with equal number of male and females actors selected picture slide was viewed for 8 seconds followed by a 5 second blank screen and central fixation point (which participants were asked to focus on between trials).

Eye-tracker Task 2. Photographs of 16 male individuals expressing happy and angry emotions were selected from the Karolinska Directed Emotional Faces database (KDEF; Lundqvist, Flykt \& Öhman, 1998). Photos were resized to 170 x 113 pixels and matrices of $16(4 x 4)$. Happy and angry faces were selected to form a happy-angry crowd type and the stimuli were adapted from a previous study conducted by Lange et al., (2011). Male faces were used in the crowd stimuli because the speed of processing male faces is quicker (Lange et al, 2011). In Task 2, we did not include neutral faces given recent discussion that people may be unbiased when viewing neutral stimuli, and previous evidence that lonely people may 
have issues with ambiguous information. Seven different crowd type ratios were created by increasing the ratio of happy to angry faces in each crowd (16 faces); 14:2 (14 happy and 2 angry), 12:4, 10:6, 8:8, 6:10, 4:12, 2:14. The task included a total of 21 slides with 3 slides per ratio trial type (i.e. 3 slides of each of the 7 ratio trial types). Participants were presented with one of two pre-randomized crowds of each slide. Each picture slide was viewed for 8 seconds followed by a 5 second blank screen and central fixation point (which participants were asked to focus on between trials). The face in the crowd paradigm was chosen for this study because participants typically show an attentional bias for a particular emotion when presented. Findings suggest an anger superiority effect for this stimuli such that participants focus their attention towards the angry faces when the crowd stimuli is overly populated with happy faces (i.e. 14 happy: 2 angry crowd type) (Lange et al, 2011).

Eye-tracker Task 3. Pictures were selected from the International affective picture system (IAPS; Lang, Bradley \& Cuthbert, 2008). Four picture types were presented at the same time on one slide depicting: (1) physical threat (violence, aggression); (2) social threat (rejection, lone individuals); (3) social positive (social interactions or social relationships); and (4) neutral (field, sky) images. Average valence ratings $(1=$ unpleasant, $9=$ pleasant $)$ from the IAPS manual of the stimuli for each picture type were as follows: (1) 3.09; (2) 3.68; (3) 7.05; (4) 7.08. An additional 18 images were included for the social threat category; they were specifically chosen to depict instances of rejection behaviour. Valence ratings for these additional images were carried out by an independent sample of 118 undergraduate students (age range 19 - 44 years; 87 females and 28 males). The additional images were classed as unpleasant $(M=3.69 ; 1=$ pleasant, $5=$ unpleasant $)$ and rated as a good example of rejecting behaviour $(M=2.49 ; 1=\operatorname{good}$ example, $5=$ weak example $)$. Some of the social rejection images had previously been used and validated in Cacioppo, Bangee et al., (2016). The study included a total of 24 slides (with 4 pictures, one each of the picture categories). Each picture 
slide was viewed for 8 seconds followed by a 5 second blank screen and central fixation point (which participants were asked to focus on between trials). The social threat pictures were chosen to show instances of social rejection or sadness and the physical threat pictures were chosen to show a threat that evokes a fear response. The social positive pictures were chosen to show positive social interactions, whilst the neutral pictures were chosen because they have been shown to produce neutral ratings. A 2 by 2 matrix was used to present the four visual scenes simultaneously to participants. Each matrix of pictures was randomized such that any of the four visual scenes could appear in any location within the 2 by 2 matrix (i.e. top left, top right, bottom left, bottom right).

\section{Data Analyses Plan}

For the analyses of data from Eye-tracker Task 1, the mean proportion of time fixating on each facial expression relative to the total captured fixation time was computed per time block across the 24 slides. Time blocks were also used to assess attention patterns over time in the analyses of Task 3 . In that analysis the mean proportion of time fixating on each picture category (social threat, physical threat, social positive, neutral) relative to the total captured fixation time was computed per time block. Time block analyses were not used to examine data for Eye-tracker Task 2 because the focus was on whether angry faces would capture the attention of lonely individuals in general and for that purpose time-blocks are not recommended (Armstrong \& Olatunji, 2012). The mean proportion of overall fixation time on the angry faces (socially threatening stimuli) relative to the total captured fixation time for each crowd ratio was computed for analysis. Also, the mean proportion of overall fixating time on the happy faces relative to the total captured fixation time for each crowd ratio was computed for analyses.

The analyses for all three tasks included linear and quadratic (curvilinear) analyses in line with previous loneliness research that used eye-tracking techniques (Bangee et al, 2014: 
Qualter et al, 2013). For the follow-up examination of significant linear and/or quadratic effects, the loneliness group was created based on the extreme upper quartile of the loneliness scores in the sample. In Tasks 1, 2, and 3 a loneliness score of 60 or above determined membership of the lonely group. That was because, according to that previous research, these extreme loneliness scorers are likely to show differences in processing compared to those scoring lower on loneliness. The data for all three tasks met the assumptions of the analyses.

Based on previous work, we expected to control for both social anxiety and depression during analyses. To determine whether that was necessary, we conducted initial correlation analyses before conducting the main set of analyses. We found that loneliness scores were correlated with the other three questionnaire measures: pearson correlations showed significant positive associations between the UCLA and the CESD $(r=.736, p<$ $.001)$, the IAS $(r=.438, p<.005)$, and BFNE $(r=.361, p<.05)$. To control for the effect of social anxiety and depression in the analyses, the three questionnaire measures using total scores (BFNE, IAS and CESD) were entered in a regression analyses to decide which measures would be used to create a standardized residual of the loneliness measure (see table $1)$.

\section{"Table 1 about here"}

Table 1 shows that CESD depression and IAS social anxiety measures were significant in the analyses. Therefore, those measures were used to form a standardized loneliness residual that controlled for depression and social anxiety in subsequent analyses.

\section{Results}

\section{Eye-Tracker Task 1 Results: Loneliness and Attention to Emotional Faces}

Linear and curvilinear analyses were conducted, with the residual of loneliness as the predictor variable, and mean proportion of fixating time on each emotional face for 8 time- 
blocks of the first four seconds viewing time (ending at 500ms, $1000 \mathrm{~ms}, 1500 \mathrm{~ms}, 2000 \mathrm{~ms}$, $2500 \mathrm{~ms}, 3000 \mathrm{~ms}, 3500 \mathrm{~ms}, 4000 \mathrm{~ms})$ and extended viewing (4500ms, 5000ms, 5500ms, $6000 \mathrm{~ms}, 6500 \mathrm{~ms}, 7000 \mathrm{~ms}, 7500 \mathrm{~ms}, 8000 \mathrm{~ms}$ ) as criterion variables. No linear or quadratic associations were found for loneliness and fixating time on the angry face during first four seconds of viewing time ( $\mathrm{L}: \beta \mathrm{s} \leq .27, p s>.05 ; \mathrm{Q}: \beta \mathrm{s} \leq .03$, $p s>.05)$ and during extended viewing (L: $\beta s \leq .27, p s>.05 ; \mathrm{Q}: \beta \mathrm{s} \leq .05, p s>.05)$, the fearful face during first four seconds of viewing time (L: $\beta \mathrm{s} \leq .08, p s>.05 ; \mathrm{Q}: \beta \mathrm{s} \leq .14, p s>.05)$ and during extended viewing (L: $\beta \mathrm{s} \leq .06, p s>.05 ; \mathrm{Q}: \beta \mathrm{s} \leq .11, p s>.05)$, the happy face during first four seconds of viewing time (L: $\beta \mathrm{s} \leq .00, p s>.05 ; \mathrm{Q}: \beta \mathrm{s} \leq .01, p s>.05)$ and during extended viewing $(\mathrm{L}: \beta \mathrm{s} \leq .01, p s$ $>.05 ; \mathrm{Q}: \beta \mathrm{s} \leq .02, p s>.05)$, the neutral face during first four seconds of viewing time (L: $\beta \mathrm{s} \leq$ $.21, p s>.05 ; \mathrm{Q}: \beta \mathrm{s} \leq-.10, p s>.05)$ and during extended viewing $(\mathrm{L}: \beta \mathrm{s} \leq .21, p s>.05 ; \mathrm{Q}: \beta \mathrm{s} \leq$ $-.09, p s>.05) .^{1}$

Chi-square analyses showed that extreme loneliness scorers and non-lonely participants were no more likely than chance to have their first fixation on the angry, fearful, happy, or neutral face $\left(\chi^{2}(3)=2.478, p=.479\right)$.

Results indicate that lonely adults do not show attention biases to negative facial expressions or towards other emotional faces. The next task expands that work to examine whether lonely adults are attentive to angry faces in a crowd of happy faces. Typically during such a task, angry faces are found to pop out (i.e. anger superiority effect) at participants.

\section{Eye-Tracker Task 2 Results: Loneliness and Attention to Anger in a Crowd of Happy}

\section{Faces}

Linear and curvilinear analyses were conducted, with the residual of loneliness as the predictor variable, and mean proportion of overall fixating time on the angry faces for the seven different ratios as criterion variables. No linear or quadratic associations were found for loneliness and overall fixation time on the angry faces in the seven crowd-types; 14 happy 
and 2 angry (L: $\beta=.19, p=.24, Q: \beta=.00, p=.98), 12$ happy and 4 angry $(\mathrm{L}: \beta=.23, p=$ $.14, \mathrm{Q}: \beta=.03, \mathrm{p}=.85), 10$ happy and 6 angry $(\mathrm{L}: \beta=.14, \mathrm{p}=.39, \mathrm{Q}: \beta=-.08, \mathrm{p}=.62), 8$ happy and 8 angry $(\beta=.23, p=.15, Q: \beta=-.07, p=.64), 6$ happy and 10 angry $(\beta=.22, p=$ $.16, \mathrm{Q}: \beta=-.03, \mathrm{p}=.85), 4$ happy and 12 angry $(\mathrm{L}: \beta=.04, \mathrm{p}=.81, \mathrm{Q}: \beta=.11, \mathrm{p}=.50), 2$ happy and 14 angry $(\mathrm{L}: \beta=.19, \mathrm{p}=.24, \mathrm{Q}: \beta=.05, \mathrm{p}=.74)$.

Furthermore, no linear or quadratic associations were found for loneliness and overall fixation time on the happy faces in the seven crowd-types; 14 happy and 2 angry (L: $\beta=-.01$, $\mathrm{p}=.96, \mathrm{Q}: \beta=-.17, \mathrm{p}=.30), 12$ happy and 4 angry $(\mathrm{L}: \beta=-.08, \mathrm{p}=.63, \mathrm{Q}: \beta=1.00, \mathrm{p}=$ .56),$,10 happy and 6 angry $(\mathrm{L}: \beta=-.08, \mathrm{p}=.63, \mathrm{Q}: \beta=.04, \mathrm{p}=.83), 8$ happy and 8 angry (L: $\beta=-.04, p=.79, Q: \beta=-.05, p=.75), 6$ happy and10 angry $(L: \beta=-.02, p=.88, Q: \beta=-.07$, $\mathrm{p}=.65), 4$ happy and 12 angry $(\mathrm{L}: \beta=.03, \mathrm{p}=.84, \mathrm{Q}: \beta=-.11, \mathrm{p}=.49), 2$ happy and 14 $\operatorname{angry}(\mathrm{L}: \beta=-.06, \mathrm{p}=.71, \mathrm{Q}: \beta=-.21, \mathrm{p}=.19) .^{2}$

Chi-square analyses showed that extreme loneliness scorers were more likely than chance to have their first fixation on an angry face in the crowd, while non-lonely participants were more likely than chance to have their first fixation on a happy face in the crowd $\left(\chi^{2}(1)\right.$ $=6.434 p=.011)$.

Results for Task 2 indicate that loneliness was not associated with overall attention to angry faces in happy crowds, with lonely adults not showing an anger superiority effect in the crowd types that were predominantly in a group of happy faces. It was the case, however, that there was a difference in initial orientation. Next, we examined whether loneliness was associated with hypervigilance to social threats that are specific to social rejection.

\section{Eye-Tracker Task 3 Results: Loneliness and Attention to Social Scenes}

Linear and curvilinear analyses were conducted, with residual of loneliness as the predictor variable, and mean proportion of fixating time on each visual scene for 8 blocks of the first four seconds viewing time (ending at 500ms, $1000 \mathrm{~ms}, 1500 \mathrm{~ms}, 2000 \mathrm{~ms}, 2500 \mathrm{~ms}$, 
$3000 \mathrm{~ms}, 3500 \mathrm{~ms}, 4000 \mathrm{~ms})$ and extended viewing $(4500 \mathrm{~ms}, 5000 \mathrm{~ms}, 5500 \mathrm{~ms}, 6000 \mathrm{~ms}$, $6500 \mathrm{~ms}, 7000 \mathrm{~ms}, 7500 \mathrm{~ms}, 8000 \mathrm{~ms}$ ) as criterion variables.

Linear, but no quadratic associations were found for loneliness and greater fixation time on the social rejection stimuli during first four seconds of viewing time ( $\mathrm{L}: \beta \mathrm{s} \geq .40, p s$ $<.005 ; \mathrm{Q}: \beta \mathrm{s} \leq .12, p s>.05)$ and during extended viewing $(\mathrm{L}: \beta \mathrm{s} \geq .39, p s<.007 ; \mathrm{Q}: \beta \mathrm{s} \leq .09$, $p s>.05)$. To examine whether extreme loneliness scorers attended differently to the social rejection stimuli, a 2 (lonely group: lonely, non-lonely) x 8 (time-blocks ending at 500ms, $1000 \mathrm{~ms}, 1500 \mathrm{~ms}, 2000 \mathrm{~ms}, 2500 \mathrm{~ms}, 3000 \mathrm{~ms}, 3500 \mathrm{~ms}, 4000 \mathrm{~ms})$ mixed measures ANCOVA with social anxiety and depression as covariates was conducted. Results showed no significant main effect of time $\left(F(3.433,133.879)=.681, p=.585, \eta p^{2}=.017\right)$. However, a significant main effect of lonely group was observed $\left(F(1,39)=16.936, p=.000, \eta p^{2}=\right.$ $.308)$, with means showing the lonely group $(M=.26)$ spent a greater amount of viewing time on the social rejection stimuli compared to the non-lonely group $(M=.18)$. No interaction effect was found for time $\mathrm{x}$ lonely group $\left(F(3.433,133.879)=.428, p=.759, \eta p^{2}=.011\right)$.

To examine whether extreme loneliness scorers attended differently to the social rejection stimuli during extended viewing, a 2 (lonely group: lonely, non-lonely) x 8 (timeblocks ending at $4500 \mathrm{~ms}, 5000 \mathrm{~ms}, 5500 \mathrm{~ms}, 6000 \mathrm{~ms}, 6500 \mathrm{~ms}, 7000 \mathrm{~ms}, 7500 \mathrm{~ms}, 8000 \mathrm{~ms})$ repeated measures ANCOVA with social anxiety and depression as covariates was conducted. Results showed no significant main effect of time $(F(3.121,121.731)=.379, p=$ $\left..776, \eta p^{2}=.010\right)$. There was a significant main effect of lonely group $(F(1,39)=16.259, p=$ $\left..000, \eta p^{2}=.294\right)$, with means showing the lonely group $(M=.25)$ spent a greater amount of viewing time on the social rejection stimuli compared to the non-lonely group $(M=.18)$. No interaction effect was found for time $\mathrm{x}$ lonely group $\left(F(3.121,121.731)=.670, p=.578, \eta p^{2}\right.$ $=.017)$. 
Similarly, linear, but no quadratic associations were found for loneliness and fixation time on the neutral stimuli during first four seconds of viewing time (L: $\beta \mathrm{s} \geq-.33$, ps <.02; $\mathrm{Q}$ : $\beta \mathrm{s} \leq .04, p s>.05)$ and during extended viewing ( $\mathrm{L}: \beta \mathrm{s} \geq-.32, p s<.02 ; \mathrm{Q}: \beta \mathrm{s} \leq .07, p s>.05)$. To examine whether extreme loneliness scorers attend differently to neutral stimuli, a 2 (lonely group: lonely, non-lonely) x 8 (time-blocks ending at $500 \mathrm{~ms}, 1000 \mathrm{~ms}, 1500 \mathrm{~ms}$, 2000ms, 2500ms, 3000ms, 3500ms, 4000ms) repeated measures ANCOVA with social anxiety and depression as covariates was conducted. Results showed no significant main effects of time $\left(F(2.193,85.514)=.886, p=.579, \eta p^{2}=.022\right)$ or lonely group $(F(1,39)=$ 2.164, $\left.p=.149, \eta \mathrm{p}^{2}=.053\right)$, and no significant time $\mathrm{x}$ lonely group interaction $(F(2.193$, $\left.85.514)=.649, p=.539, \eta p^{2}=.016\right)$.

To examine whether extreme loneliness scorers attended differently to neutral stimuli during extended viewing, a 2 (lonely group: lonely, non-lonely) x 8 (time-blocks ending at $4500 \mathrm{~ms}, 5000 \mathrm{~ms}, 5500 \mathrm{~ms}, 6000 \mathrm{~ms}, 6500 \mathrm{~ms}, 7000 \mathrm{~ms}, 7500 \mathrm{~ms}, 8000 \mathrm{~ms})$ repeated measures ANCOVA with social anxiety and depression was conducted. Results showed no significant main effects of time $\left(F(3.096,120.725)=.081, p=.973, \eta p^{2}=.002\right)$ or lonely group $(F(1$, $\left.39)=2.099, p=.155, \eta p^{2}=.051\right)$, and no significant time $\mathrm{x}$ lonely group interaction $(F$ $\left.(3.096,120.725)=.950, p=.421, \eta p^{2}=.024\right)$.

No linear or quadratic associations were found for loneliness and fixating time on the physical threat stimuli during first four seconds of viewing time (L: $\beta \mathrm{s} \leq .16, p s>.05$; $\mathrm{Q}: \beta \mathrm{s} \leq$ $-.26, p s>.05)$ and during extended viewing (L: $\beta \mathrm{s} \leq .19, p s>.05$; $\mathrm{Q}: \beta \mathrm{s} \leq-.29, p s>.05)$, nor on the social positive stimuli during the first four seconds of viewing time (L: $\beta \mathrm{s} \leq-.07, p s$ $>.05 ; \mathrm{Q}: \beta \mathrm{s} \leq-.19, p s>.05)$ and during extended viewing $(\mathrm{L}: \beta \mathrm{s} \leq-.08, p s>.05 ; \mathrm{Q}: \beta \mathrm{s} \leq-.20$, ps $>.05) .^{3}$

Chi-square analyses showed that extreme loneliness scorers and non-lonely participants were no more likely than chance to have their first fixation on the social 
rejection, physical threat, social positive, or neutral images $\left(\chi^{2}(1)=1.381, p=.240\right)$. Interestingly, the majority of participants in the study had their first fixation on the social rejection stimuli with only a few participants having their first fixation on the social positive image.

Results from Task 3 showed that loneliness was associated with viewing social rejection images for a greater duration across time. Thus, it seems lonely adults were more attentive to social threats linked to social rejection. Furthermore, the effect of loneliness was associated with less viewing time of neutral images across time; loneliness was not found to be associated with viewing the social positive and physical threat images.

\section{Discussion}

The current study directly examined the hypervigilance to social threat hypothesis proposed by Cacioppo and Hawkley (2009) and adapted by Qualter et al., (2015), assessing whether attention to social threats was a generalized or specific bias among lonely people. Findings showed that loneliness was not associated with increased attention to social threats depicted as negative facial expressions (i.e. anger), was not associated with attention biases to social threats displayed in a crowd of happy faces (i.e. anger superiority effect), but was associated with an initial orientation to angry faces rather than happy faces, and more attention being paid to images of social rejection. Findings suggest that lonely people in the current study were sensitive to (1) initial presentation of angry faces in crowds, and (2) social threats linked specifically to social rejection as depicted in social scenes, tending to maintain their attention to those stimuli over an extended viewing time. Those findings support those from previous eye-tracking work (Qualter et al, 2013), in which lonely children found it difficult to disengage from socially threatening stimuli in the form of rejection threats, and evidence that lonely young adults showed initial vigilance to socially threatening video footage (Bangee et al, 2014). The findings from Task 1 yielded similar non-significant 
associations for loneliness and attention to emotional faces in line with findings by Lodder et al (2015), suggesting that loneliness is not related to specific viewing patterns of facial expressions. Findings from Task 2 support Lodder et al (2016) and Lodder et al., (2015), showing that loneliness is associated with an initial orientation towards angry faces, but not sustained attention towards that stimuli over viewing time.

\section{Theoretical Implications of Findings}

Findings support the current model of loneliness (Cacioppo and Hawkley, 2009; Qualter et al., 2015), where it is argued loneliness is associated with a negative cognitive bias that influences attention, interpretation, and memory related to stimuli that signal rejection or social isolation. The thesis is that loneliness drives people to reconnect, but, in that process, they must avoid others who might reject them; people respond to their loneliness with reconnecting behaviour, but also avoidance of rejection, which would cause further psychological harm and discomfort (Qualter et al., 2015). Our findings support that thesis providing evidence in support of the proposal that we avoid rejection when lonely and protect ourselves from social harm: current findings suggest that lonely people are more like to attend to social rejection/exclusion in the environment when there is meaningful information outside the face that provides visual contextual information, perhaps in the form of body postures, gestures, or other contrasting positive social information. Future work will want to explore contextual information further, perhaps examining the role of auditory information, and whether it provides further contextual information that lonely people will use in their assessment of threat.

\section{Practical Implications of Findings}

We found that lonely adults are more attentive to social threats that are linked to contextual social rejection. While attention to potential rejection from the group is good for survival, enabling the avoidance of social groups/individuals that may further reject us, such 
attentiveness may be problematic in the long run because it means we avoid potentially positive social relationships because we interpret them as dangerous, or because we are too focused on the potential threats in the environment. Thus, interventions for people who experience prolonged loneliness could include a re-focusing of attention to address this specific cognitive bias where it has become problematic, supporting lonely adults to re-frame situations that they view as socially rejecting and teaching them the skills to relocate attention from social threats (Qualter et al, 2013; Qualter et al., 2015). Such attention re-training in the treatment of anxiety, designed to help people move their attention away from threat, have been shown to have moderate and significant effects in reducing anxiety (Shechner \& BarHaim, 2016). Future work will want to determine whether those attention re-training programs could be successfully used with people suffering from prolonged loneliness.

Our findings also indicate that cognitive-behavioural strategies would best support those who score high on loneliness because those individuals were found to have the most difficulty in disengaging from social threat; that group should be the primary focus for any interventions proposed. While we recommend such an approach to intervention, we are also mindful that research is needed to examine whether the cognitive bias causes behavioural deficiencies; with such prospective work, more effective interventions can be developed.

\section{Conclusion}

Findings from the current set of eye-tracker tasks suggest that lonely people are sensitive to social threats linked specifically to social rejection, tending to maintain their attention to those stimuli. Such findings have implications for intervention, with recommendations that programmes should focus on attention re-training and the re-framing of situations that lonely people view as socially threatening, specifically those they see as signalling social rejection or social isolation. 
Acknowledgments: We wish to thank Rebecca Nowland, University of Manchester, for her help with the data collection. 


\section{References}

Armstrong, T., \& Olatunji, B. O. (2012). Eye tracking of attention in the affective disorders: A metaanalytic review and synthesis. Clinical Psychology Review, 32, 704-723. doi:10.1016/j.cpr.2012.09.004

Bangee, M., Harris, R. A., Bridges, N., Rotenberg, K. J., \& Qualter, P. (2014). Loneliness and attention to social threat in young adults: Findings from an eye-tracker study. Personality and Individual Differences, 63, 16-23. doi:10.1016/j.paid.2014.01.039.

Bögels, S. M., \& Mansell, W. (2004). Attention processes in the maintenance and treatment of social phobia: hypervigilance, avoidance and self-focused attention. Clinical psychology review, 24(7), 827-856. doi:10.1016/j.cpr.2004.06.005

Buckner, J.D., Maner, J.K., \& Schmidt, N.B. (2010). Difficulty disengaging attention from social threat in social anxiety. Cognitive Therapy Research, 34, 99-105. doi:10.1007/s10608-008-9205y

Burklund, L. J., Eisenberger, N. I., \& Lieberman, M. D. (2007). The face of rejection: Rejection sensitivity moderates dorsal anterior cingulate activity to disapproving facial expressions. Social Neuroscience, 2(3-4), 238-253. http://doi.org/10.1080/17470910701391711

Cacioppo, S., Balogh, S., \& Cacioppo, J. T. (2015). Implicit attention to negative social, in contrast to nonsocial, words in the Stroop task differs between individuals high and low in loneliness: evidence from event-related brain microstates. Cortex, 70, 213-233. doi:10.1016/j.cortex.2015.05.032

Cacioppo, S., Bangee, M., Balogh, S., Cardenas-Iniguez, C., Qualter, P., \& Cacioppo, J. T. (2016). Loneliness and implicit attention to social threat: A high-performance electrical neuroimaging study. Cognitive neuroscience, 7(1-4), 138-159. doi:10.1080/17588928.2015.1070136

Cacioppo, J.T., Ernst, J. M., Burleston, M. H., McClintock, M. K., Malarkey, W. B., Hawkley, L. C., Kowalewski, R. B., Paulsen, A., Hobson, A., Hugdahl, K., Spiegel, D., \& Bernston, G. G. 
(2000). Lonely traits and concomitant physiological processes: the MacArthur social neuroscience studies. International Journal of Psychophysiology, 35, 143-154. doi:10.1016/S0167-8760(99)00049-5

Cacioppo, J.T., \& Hawkley, L.C. (2009). Perceived social isolation and cognition. Trends in Cognitive Science, 13, 447-454. doi:10.1016/j.tics.2009.06.005

Cacioppo, J. T., Hawkley, L. C., \& Thisted, R. A. (2010). Perceived social isolation makes me sad: 5year cross-lagged analyses of loneliness and depressive symptomatology in the Chicago Health, Aging, and Social Relations Study. Psychology and aging, 25(2), 453. doi:10.1037/a0017216

Cacioppo, J.T., Norris, C.J., Decety, J., Monteleone, G., \& Nusbaum, H. (2009). In the eye of the beholder: Individual differences in perceived social isolation predict regional brain activation to social stimuli. Journal of Cognitive Neuroscience, 21, 83-92. doi:10.1162/jocn.2009.21007

Caseras, X., Garner, M., Bradley, B. P., \& Mogg, K. (2007). Biases in visual orienting to negative and positive scenes in dysphoria: An eye movement study. Journal of abnormal psychology, 116(3), 491-497. doi:10.1037/0021-843X.116.3.491

Cisler, J. M., \& Koster, E. H. (2010). Mechanisms of attentional biases towards threat in the anxiety disorders: An integrative review. Clinical Psychology Review, 30, 203-216. doi: 10.1016/j.cpr.2009.11.003

Doane, L. H. \& Adam, E. K. (2010). Loneliness and cortisol: Momentary, day-to-day, and trait associations. Psychoneuroendocriniology, 35, 430-441. doi:10.1016/j.psyneuen.2009.08.005

Ebner, N. C., He, Y. I., \& Johnson, M. K. (2011). Age and emotion affect how we look at a face: Visual scan patterns differ for own-age versus other-age emotional faces. Cognition \& emotion, 25(6), 983-997. doi:10.1080/02699931.2010.540817

Egidi, G., Shintel, H., Nusbaum, H.C., \& Cacioppo, J.T. (2008). Social isolation and neural correlates of attention control. 20th Annual Meeting of the Association for Psychological Science; Chicago, IL. 
Fox, E., Russo, R., \& Dutton, K. (2002). Attentional bias for threat: Evidence for delayed disengagement from emotional faces. Cognition \& Emotion, 16(3), 355-379. doi:10.1080/02699930143000527

Gardner, W.L., Pickett, C.L., Jefferis, V., \& Knowles, M. (2005). On the outside looking in: Loneliness and social monitoring. Personality and Social Psychology Bulletin, 31, 15491560. doi:10.1177/0146167205277208

Garner, M., Mogg, K., \& Bradley, B.P. (2006). Orienting and Maintenance of Gaze to Facial Expressions in Social Anxiety. Journal of Abnormal Psychology, 115, 760-770. doi:10.1037/0021-843X.115.4.760

Goeleven, E., De Raedt, R., Leyman, L., \& Verschuere, B. (2008). The Karolinska Directed Emotional Faces: A validation study. Cognition and Emotion, 22(6), 1094-1118.

Griffin, J. (2010). The lonely society. London: Mental Health Foundation.

Hartshorne, J. K. \& Germine, L. T. (2015). When does cognitive functioning peak? The asynchronous rise and fall of different cognitive abilities across the life span. Psychological Science, 26, 433-443. doi:10.1177/0956797614567339

Heinrich, L.M., \& Gullone, E. (2006). The clinical significance of loneliness: A literature review. Clinical Psychology Review, 26, 695-718. doi:10.1016/j.cpr.2006.04.002

Hermans, D., Vansteenwegen, D., \& Eelen, P. (1999). Eye movement registration as a continuous index of attention deployment: Data from a group of spider anxious students. Cognition and Emotion, 13, 419-434. doi:10.1080/026999399379249

Holt-Lunstad, J., Robles, T. F., \& Sbarra, D. A. (2017). Advancing social connection as a public health priority in the United States. American Psychologist, 72, 517530. http://dx.doi.org/10.1037/amp0000103

Jones, W. H., Freemon, J. E., \& Goswick, R. A. (1981). The persistence of loneliness: 
Self and other determinants. Journal of Personality, 49, 27-48. doi:10.1111/j.14676494.1981.tb00844.x

Jones, W. H., Rose, J., \& Russell, D. W. (1990). Loneliness and social anxiety.

Handbook of social and evaluation anxiety. Leitenberg, Harold (Ed); pp. 247-266. New York, NY, US: Plenum Press.

Kanai, R., Bahrami, B., Duchaine, B., Janik, A., Banissy, M. J., \& Rees, G. (2012). Brain structure links loneliness to social perception. Current Biology, 22(20), 1975-1979. doi:10.1016/j.cub.2012.08.045

Knowles, M. L., Lucas, G. M., Baumeister, R. F., \& Gardner, W. L. (2015). Choking Under Social Pressure Social Monitoring Among the Lonely. Personality and Social Psychology Bulletin, 41(6), 805-821. doi:10.1177/0146167215580775

Lang, P. J., Bradley, M. M., \& Cuthbert, B. N. (2008). International affective picture system (IAPS): Affective ratings of pictures and instruction manual. Technical report A-8.

Lange, W.G., Heuer, K., Langner, O., Keijsers, G.P.J., Becker, E.S., \& Rinck, M. (2011). Face value: Eye movements and the evaluation of facial crowds in social anxiety. Journal of Behavioral Therapy \& Experimental Psychiatry, 42, 355-363. doi:10.1016/j.jbtep.2011.02.007

Lasgaard, M., Goossens, L., \& Elklit, A. (2011). Loneliness, depressive symptomatology, and suicide ideation in adolescence: Cross-sectional and longitudinal analyses. Journal of abnormal child psychology, 39(1), 137-150. doi:10.1007/s10802-010-9442-х

Lazerus, T., Ingbretsen, Z. A., Stolier, R. M., Freeman, J. B., \& Cikara, M. (2016). Positivity bias in judging ingroup members' emotional expressions. Emotion, 16(8), 11171125. doi: 10.1037/emo0000227

Leary, M. R. (1983a). A brief version of the Fear of Negative Evaluation Scale. Personality and Social Psychology Bulletin, 9, 371-376. doi: 10.1177/0146167283093007 
Leary, M. R. (1983b). Social anxiousness: The construct and measurement. Journal of Personality Assessment, 47, 66-75.doi: 10.1207/s15327752jpa4701_8

Leary, M. R., \& Kowalski, R. M. [1993]. The Interaction Anxiousness Scale: Construct and criterion-related validity. Journal of Personality Assessment, 61, 136-146. doi: 10.1207/s15327752jpa6101_10

Lim, M, H., Rodebaugh, T, L., Zyphur, M, J., \& Gleeson, J, F. (2016) Loneliness over time: The crucial role of social anxiety. Journal of Abnormal Psychology. Vol. 125, 620-630, doi:10.1037/abn0000162

Lodder, G. M., Scholte, R. H., Clemens, I. A., Engels, R. C., Goossens, L., \& Verhagen, M. (2015). Loneliness and hypervigilance to social cues in females: An eye-tracking study. PloS one, 10(4), e0125141. doi:10.1371/journal.pone.0125141

Lodder, G. M. A., Scholte, R. H. J., Goossens, L., Engels, R. C. M. E., \& Verhagen, M. (2016). Loneliness and the social monitoring system: Emotion recognition and eye gaze in a real-life conversation. British Journal of Psychology, 107(1), 135-153. doi: 10.1111/bjop.12131

Lundqvist, D., Flykt, A., \& Öhman, A. (1998). The Karolinska directed emotional faces (KDEF). CD ROM from Department of Clinical Neuroscience, Psychology section, Karolinska Institutet, 91-630.

Mahon, N. E., Yarcheski, A., Yarcheski, T. J., Cannella, B. L., \& Hanks, M. M. (2006). A meta-analytic study of predictors for loneliness during adolescence. Nursing research, 55(5), 308-315. doi:10.1097/00006199-200609000-00003

Mogg, K., Bradley, B. P., De Bono, J., \& Painter, M. (1997). Time course of attentional bias for threat information in non-clinical anxiety. Behaviour research and therapy, 35(4), 297-303. doi:10.1016/S0005-7967(96)00109-X 
Munoz, D. P., Broughton, J. R., Goldring, J. E., \& Armstrong, I. T. (1998). Age-related performance of human subjects on saccadic eye movement tasks. Experimental Brain Research, 121(4), 391-400. doi:10.1007/s002210050473

Peplau, L. \& Perlman, D. (1982). Perspectives on loneliness. In L. Peplau, \& D. Perlman (Eds.). Loneliness: A Sourcebook of Current Theory, Research and Therapy. New York: John Wiley and Sons.

Qualter, P., Rotenberg, K.J., Barrett, L., Henzi, P., Barlow, A., Stylianou, M.S., \& Harris, R.A. (2013). Investigating Hypervigilance for Social Threat of Lonely Children. Journal of Abnormal Child Psychology, 41, 325-338. doi:10.1007/s10802-012-9676-X

Qualter, P., Vanhalst, J., Harris, R., Van Roekel, E., Lodder, G., Bangee, M., ... \& Verhagen, M. (2015). Loneliness across the life span. Perspectives on Psychological Science, 10(2), 250264. doi:10.1177/1745691615568999

Radloff, L. S. (1977). The CES-D scale a self-report depression scale for research in the general population. Applied psychological measurement, 1(3), 385-401. doi:10.1177/014662167700100306

Russell, D. (1996). UCLA Loneliness Scale (version 3): Reliability, validity, and factor structure. Journal of Personality Assessment, 66, 20-40.

Schofield, C. A., Johnson, A. L., Inhoff, A. W., \& Coles, M. E. (2012). Social anxiety and difficulty disengaging threat: Evidence from eye-tracking. Cognition \& emotion, 26(2), 300311. doi:10.1080/02699931.2011.602050

Sears, C. R., Thomas, C. L., LeHuquet, J. M., \& Johnson, J. C. (2010). Attentional biases in dysphoria: An eye-tracking study of the allocation and disengagement of attention. Cognition and Emotion, 24(8), 1349-1368. doi:10.1080/02699930903399319 
Snodgrass, M. A. (1987). The relationships of differential loneliness, intimacy, and characterological attributional style to duration of loneliness. Journal of Social Behavior and Personality, 2(2), 173.

Spithoven, A.W. M., Bijttebier, P., \& Goossens, L. (2017). It is all in their mind: A review on information processing bias in lonely individuals. Clinical Psychology Review, 58, 97-114. doi: 10.1016/j.cpr.2017.10.003

Suess, F., Rabovsky, M., \& Rahman, R. A. (2015). Perceiving emotions in neutral faces: expression processing is biased by affective person knowledge. Social, Cognitive, Affective Neuroscience, 10, 531-536. doi: 10.1093/scan/nsu088

Vanhalst, J., Gibb, B. E., \& Prinstein, M. J. (2015). Lonely adolescents exhibit heightened sensitivity for facial cues of emotion. Cognition and emotion, 1-7. doi:10.1080/02699931.2015.1092420

Verhaeghen, P., \& Salthouse, T. A. (1997). Meta-analyses of age-cognition relations in adulthood: Estimates of linear and nonlinear age effects and structural models. Psychological bulletin, 122(3), 231. doi:10.1037/0033-2909.122.3.231

Weiss, E. M., Kemmler, G., Deisenhammer, E. A., Fleischhacker, W. W., \& Delazer, M. (2003). Sex differences in cognitive functions. Personality and individual differences, 35(4), 863875. doi:10.1016/S0191-8869(02)00288-X 
Table 1

Table 1: Questionnaire measures entered into the analyses to create the loneliness residual

\begin{tabular}{lcc} 
Questionnaire measure & Beta value & p value \\
\hline CESD (depression) & .680 & .000 \\
BFNE (social anxiety) & -.050 & .697 \\
IAS (social anxiety) & .302 & .018 \\
\hline
\end{tabular}




\section{Footnotes}

${ }^{1}$ Pre-analyses with loneliness (not controlling for social anxiety and depression) found significant associations between loneliness and attention to angry and neutral faces.

${ }^{2}$ Pre-analyses with loneliness (not controlling for social anxiety and depression) found significant associations between loneliness and attention to the angry faces in predominantly crowds of happy faces.

${ }^{3}$ Pre-analyses found loneliness (not controlling for social anxiety and depression) was associated with greater amount of viewing time on the social threat stimuli, trends for physical threat, and less amount of viewing time on the neutral images. 